\title{
Denominación de origen de cacao ecuatoriano: ¿un aporte de marketing global?
}

\section{Denomination of origin of Ecuadorian cocoa: a contribution of global marketing?}

María Dolores Quintana Lombeida

Universidad Tecnológica Equinoccial, Ecuador

Jairo Vladimir Aguilar Herrera

Culinary Trainer School, Ecuador

Autor para correspondencia: maria.quintana@ute.edu.ec, chefjairoaguilar@gmail.com

Fecha de recepción: 23 de agosto de 2018 - Fecha de aceptación: 01 octubre de 2018

Resumen: La presente investigación radica en la importancia del cacao fino de aroma ecuatoriano que simboliza el $60 \%$ del mercado a nivel mundial. A través de un estudio descriptivocorrelacional, multivariado e instrumentos de recolección aplicados como entrevistas se evidenció las características del cacao ecuatoriano, cotizado por sus excelentes notas florales base para la elaboración de subproductos en Europa, Norteamérica y Asia. Con la intención de incentivar el comercio internacional, el Instituto Ecuatoriano de Propiedad Intelectual (IEPI), prescribió la denominación de origen "Cacao Arriba" que permitió su certificación para todo el cacao fino de aroma que procediera del territorio nacional. Entre los resultados hallados se identificó que en el caso de Ecuador la "zona arriba", reconocida desde la época de la colonia, representa a la Cuenca baja del rio Guayas que conforman las provincias de Los Ríos y Guayas, la cual posee características particulares por la influencia climática, la corriente de Humboldt y El Niño. Sin embargo la denominación de origen en el Ecuador se expone con la finalidad de promoción como un fruto con garantía de procedencia que contradice las características propias de discriminación geográfica que son específicas de una zona estrictamente delimitada, su impacto entre los agricultores no trascendió, mientras que para el mercado internacional no es una evidencia de características organolépticas que garanticen el origen, características y calidad específico del producto; por lo tantobajo esos parametros no representa una estrategia de marketing global.

Palabras Claves: marketing global; denominación de origen; cacao arriba; cacao fino de aroma

Abstract: The present investigation is based on the importance of fine cocoa with an Ecuadorian aroma that symbolizes $60 \%$ of the market worldwide. Through a descriptive-correlational, multivariate study and the collection of applications such as interviews, the characteristics of organic cocoa are evidenced, quoted for its excellent base notes of flowers for the production of by-products in Europe, North America and Asia. With the intention of encouraging international trade, the Ecuadorian Institute of Intellectual Property (IEPI), in 2008, prescribed the designation of origin "Cacao Arriba" unfortunately its certification was sent for all the fine aroma cocoa that came from the national territory. Among the results found, it was identified that in the case of Ecuador, the "upstream zone", recognized since colonial times, represents the Lower Guayas River 
Basin that make up the provinces of Los Ríos and Guayas, which has particular characteristics the climatic influence, the current of Humboldt and El Niño. However, the denomination of origin in Ecuador is set out for the purpose of promotion, with the guarantee that it contradicts the characteristics of geographical discrimination, which is specific to a strictly delimited area, its impact among farmers did not extend, while for the international market it is not an evidence of organoleptic characteristics that guarantee the origin, characteristics and specific quality of the product; therefore, it does not represent a global marketing

Key Words: comercialización global; denominación de origen; cacao arriba; cacao fino de aroma

\section{Introducción}

Durante varios años el cacao fue un símbolo de auge económico en el Ecuador, sus máximos representantes fueron las provincias de Los Ríos, Guayas y Manabí; sin embargo esta suma del terruño que guardaba el país desembocaba en un elemento tras otro que lo conectó con Europa y otros países altamente industrializados, con sed de las cualidades únicas; tanto florales y frutales que poseía el cacao de la tierra ecuatoriana.

La FAO en el año 2011 desarrolló el proyecto Calidad de los Alimentos Vinculada al Origen y las Tradiciones en América Latina; en el cual se planteó que ocho países tenían pertinencia con criterios de Denominación de Origen donde se identifica una zona geográfica estrictamente delimitada con la finalidad de potencializar productos de alta calidad y cualidades únicas prioritariamente por; ranking, tipicidad, tradición e historia del género. Ecuador en el año 2011 a través del Instituto Ecuatoriano de Propiedad Intelectual, se suscribió a dicho proyecto con cacao y se obtuvo la denominación de origen para la variedad de cacao fino de aroma, uno de los productos más representativos del país.

A través de este documento se analiza la denominación de origen planteada en el Ecuador y, si esta posee las características necesarias para impulsar el desarrollo e incrementar la representatividad del cacao en el mercado local e internacional.

\section{Desarrollo}

\section{La historia cacaotera ecuatoriana}

Los factores de comercio y mercado internacional fomentaron en la década de 1770 las exportaciones, en un ambiente donde el capital y la mano de obra eran escasos. El valor de las exportaciones de cacao creció ininterrumpidamente, desde mediados del siglo XVIII hasta la crisis de 1811 pasaron de un valor aproximado de cien mil pesos, hasta cerca del medio millón. (Contreras, 1994). Por lo tanto desde 1860 y hasta 1920 el Ecuador tuvo su primer boom cacaotero.

Sin embargo las condiciones de productividad y comercialización del período, convirtieron a grandes zonas de monocultivo en el resultado de limitados procesos administrativos que confluyeron en la enfermedad Escoba de Bruja, Según (Porras \& Sánchez, 1991) considerada como la más dañina para el cultivo de cacao, a pesar de que el nivel de severidad varía de acuerdo con las condiciones de clima, presión, variedad de cacao y manejo de 
la plantación, las pérdidas pueden alterar hasta el 70\% de la producción. El primer brote de esta enfermedad fue registrado en Surinam en el año 1985.

Ecuador no estuvo preparado para determinar estrategias que lo contrarresten con enfermedades en su agricultura, En 1920 la enfermedad escoba de bruja acabo con los cultivos de cacao en el Ecuador lo que destruiría una economía mono productiva, esclavitud, trabajo infantil y una fugaz pérdida del mayor sitial de mercado, en solo una década perdió 50 años de alto posicionamiento en el mercado mundial. Es entonces cuando Ghana empieza a posicionarse con un producto de menor calidad.

Entendiéndose que el cacao nacional tiene características organolépticas que marcan la representatividad del $56 \%$ del mercado mundial especialmente por las condiciones del terreno o terruño donde se suman las del; suelo, tradiciones, talento humano y clima del Ecuador. Tras décadas en las mismas condiciones, a partir del año 2003 el país tuvo un incremento del ciento diez por ciento en exportación de cacao, entre los principales factores de éxito se resaltan podas impulsadas por el Ministerio de Agricultura y Ganadería a nivel Nacional en una articulación publico privada, los precios internacionales, y la representatividad que posee el Ecuador como el primer proveedor mundial del cacao fino de aroma y de cacao arriba, con una caracteristicacmazorca de color amarillo. (Anecacao)

Sin embargo existen variedades con mejor calidad en zonas específicas y altamente demandadas en; Los Ríos, Guayas y Manabí presentan cualidades frutales y florales altamente marcadas. De tal manera que por todas estas bondades son exclusivamente tres provincias que no lo cultivan

Para el año 2000 Ecuador arranca con su nuevo boom cacaotero, vinculándose con un proceso de expansión e marca con varias empresas, entre estas empresas se reconocen.

Tabla N. 1 mayores Empresas productoras de chocolate con cacao fino de aroma en Ecuador

\begin{tabular}{ll}
\hline Empresa & Datos generales \\
\hline Pacari & Inicia en el 2002. Con elementos de responsabilidad social y del \\
comercio justo, produce con otros productos ecuatorianos \\
principalmente andinos, ha ingresado a mercados \\
internacionales principalmente estados unidos, Asia y mercado \\
judío con sus certificaciones de calidad como estrategias de \\
dirección comercial
\end{tabular}




\begin{tabular}{cl}
\hline Kallari & $\begin{array}{l}\text { Inicio en el año } 2000 \\
850 \text { socios de la comunidad Kichwa del Napo, productores } \\
\text { orgánicos y artesanales con cacao fino de aroma }\end{array}$ \\
\hline Elaborado por autores.
\end{tabular}

\title{
Marketing global
}

El marketing global o internacional es la realización de actividades de negocios diseñadas para planear, cotizar, promover y dirigir el flujo de bienes y servicios de una compañía hacia los consumidores o usuarios de más de una nación para obtener un beneficio. (Cateora \& Graham, 1999, p.8)

Las características primordiales del marketing global radican en consolidar a las estrategias de dirección comercial alineadas a nuevos mercados entendiéndose que cada país posee características propias, elementos de cultura, tradiciones, intereses, hábitos y, psicología de consumo conforme a cada su realidad. Toda esta suma de variables hace que las empresas deban analizar al consumidor, variables endógenas y exógenas antes de trascender fronteras con sus productos, en el caso que estos hayan cruzado limites internacionales, se deberán realizar planes estratégicos para su mejora, permanencia o reposicionamiento en los diferentes demandantes.

Conforme los mercadólogos los elementos en mercados internacionales tendrán más factores incontrolables que dificultan su operatividad desde el país de origen, a continuación se describen algunos de ellos

TablaN.1 Elementos exógenos en el marketing global

\author{
Elementos \\ Barreras comerciales y arancelarias \\ Cambio monetario \\ Estándares de salud,seguridad,y calidad del producto \\ Tecnología \\ Geografía, clima, elementos naturales \\ Seguridad Social \\ Responsabilidad social y ambiental \\ Política \\ Disponibilidad de recursos, vialidad, servicios básicos \\ Población \\ Cultura, religión \\ Elaborado por autores. Marketing Internacional (Cateora \& Graham, 1999)

\section{Métodos} \\ La presente investigación fue de carácter descriptivo, analítico, correlacional incluyo \\ técnicas como; observación en empresas afines al comercio de chocolate fino de aroma, \\ entrevistas a expertos en el Secretaria Nacional de Propiedad Intelectual (SENADI) en Ecuador, \\ agricultores y productores de chocolate y subproductos. Se constituyó en multivariado al \\ interrelacionar al cacao como producto único en el país con la variable de influencia \\ internacional y la prescripción de la denominación de origen para cacao arriba ecuatoriano.
}

\section{Denominación de origen}


Una denominación de origen según (Millán, 2008) se identifica de la misma manera que una marca, se establece como una herramienta para diferenciar los bienes en atención a su origen geográfico tal como la marca expone un origen empresarial. Una denominación de origen adecuadamente planteada y circunscrita en límites geográficos permitirá identificar las zonas específicas donde el país genera las mejores variedades de cacao nacional; adicionalmente establecerá beneficiarios en toda la cadena de valor desde el centenar de pequeños agricultores que se dedica a esta actividad hasta los comerciantes y consumidores de un producto con una etiqueta que asegure la identidad de un producto en todo su enlace productiva.

Las denominaciones de origen nacen con el interés de fabricar productos que respondan a un origen y que se respete su nombre para esa zona en específico. "Se considera que la denominación de origen más antigua en el mundo se concedió en 1887 al Sindicato de los Grandes Marqueses de Champagne, cuando Francia reconoció la propiedad exclusiva del nombre Champagne para los vinos espumosos de esa región" (Errázuriz, 2010). Esa primera bebida espumosa resulto del enfrentamiento entre cultivadores y vinicultores que se unieron para proteger la imitación del producto en otras zonas del mundo, este grupo exigía que el estado Francés declarara el uso del nombre "champan" exclusivamente para el producto que se originara de esta zona, ya que mencionaban que ninguno poseía características similares en ninguna parte del mundo.

Dicho grupo de profesionales en vinicultura consideraban que la creación de un producto único como el champán no era simplemente cuestión de seguir el conocimiento de las técnicas de fermentación y almacenamiento que se habían desarrollado generación tras generación en la región. Este proceso de construcción de la distinción del champán descansaba, además, en una cuidadosa selección de los insumos para producir dicha bebida así como de las condiciones del suelo, del aire y del clima que se debían observar. Fue por ello que, después de años de selección y acomodos, los productores de Champagne acordaron que serían sólo tres variedades de uva las que podían usarse en la elaboración de este vino espumoso específico (Rodríguez, 2004).

Particularmente las denominaciones de origen son planteamientos que se han generado en diversos productos en varias partes del mundo han tenido éxito con productos de diferente índole han trascendido fronteras a través del marketing global, por su buen manejo de marca, su impacto por un buen producto y su respeto al productor y el espacio geográfico tal como las diferentes perspectivas teóricas lo afirman.

En la Unión Europea se han desarrollado miles de denominaciones de origen, solo en España existen y cuyo impacto ha sido evidenciado en el ámbito económico, puesto que se denota la calidad y representatividad de éste, y fortalece la economía de todos los actores, además se suman otras iniciativas como el comercio justo y varios sellos de calidad que cada vez van tomando mayor impulso en el posicionamiento en el mercado internacional y el consumidor local.

En América Latina ya se encuentra países con estos modelos, los cuales han sido bastante eficaces, tal es el caso de México Figura N.1, que presenta 15 denominaciones de origen en productos como bebidas alcohólicas, café, artesanías y productos agrícolas. 


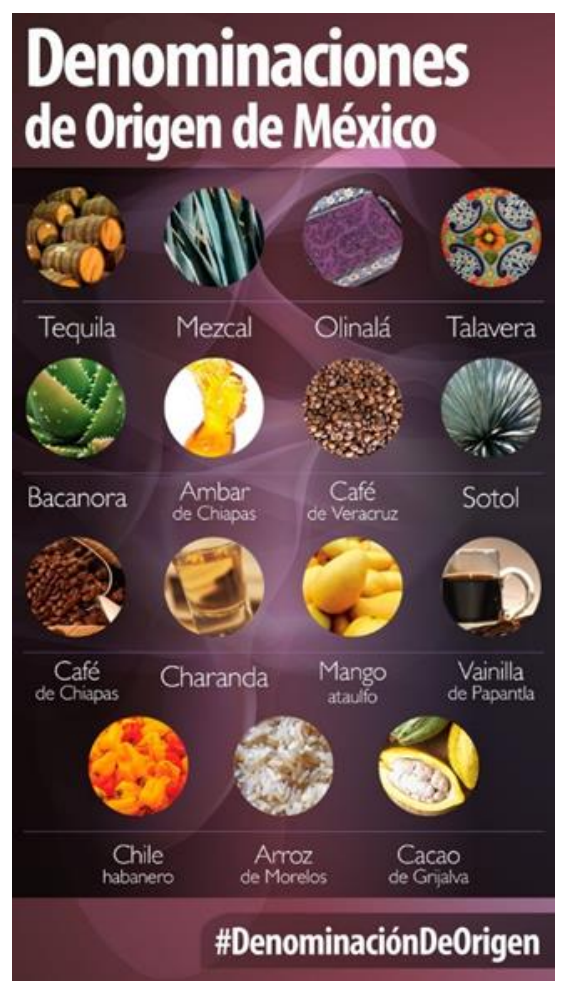

Figura 1. Denominaciones de origen Mexicanas

Fuente: Secretaria de Economía. gob.mx/se

Mientras que la Unión Europea cuenta con 2000 denominaciones de origen en; cerveza, vino, quesos, especias, agrícolas, grasas y otros.

Los objetivos principales que se propone en esta investigación radican en la estructura y valoración de la denominación de origen de otros productos alimenticios en el mundo. En este sentido, se ha observado que a pesar de la tendencia a la estandarización de los productos agroalimentarios, un importante y creciente sector de los consumidores prefieren los productos artesanales, locales, de sabores y olores particulares y que encierran en sí mismos la tradición del territorio donde se originaron (Ilbery B, 2000)

La biodiversidad de las zonas ha provocado la diferenciación de productos agrícolas y especialmente vinícolas en él, entre los que se citan: vino de jerez, champagne, cerveza de Dortmund. Por otra parte también se lo aplica a productos artesanales: sombreros de paja toquilla recientemente en Ecuador, porcelana de Limoges, Aceros de Solingen, vinos de Mendoza, Argentina.

\section{Denominación de origen en el Ecuador}

La estructura de la denominación de origen se la nombró Cacao Arriba y se elaboró conforme a las técnicas de; trabajo de campo y encuestas a agricultores del sector cacaotero de todo el país por parte de dos ingenieros agrónomos.

Posteriormente la conjugación del documento fue conformado por un panel de expertos adscritos a organismos estatales del sector agrícola, de propiedad intelectual y exportador. 
Para así describir convenientemente las características físicas de la almendra en estado fermentado y seco proveniente de la especie The obroma cacao donde se destacan como característica egocéntrica, su sabor floral.

Para la adscripción a la denominación de origen, posteriormente se toman las premisas de; factores naturales y humanos, topográficos, del cacao Arriba, fino de aroma, sabor floral y delimitación Geográfica:

\section{Factores topográficos:}

Factores agroclimáticos: se delimita la zona geográfica de todo el territorio ecuatoriano, con precipitaciones de 2000 a 4000mm y que se cultivan en temperatura media de $24 \mathrm{C}$, la zona productora del cacao arriba posee un clima tropical ecuatorial.

Factores topográficos: se mencionan cualidades de la zona costera así como de la amazonia con escasas pendientes, estribaciones en las cordilleras hasta cuando alcanza los 400 msnm. El suelo en la amazonia son aluviales y con sedimentos volcánicos.

Factores del cacao Arriba, fino de aroma, sabor floral:

Mazorcas: se la describe como amarilla al madurar y usualmente con diez surcos

Flor: se manifiesta a esta variedad se distingue del resto del mundo por el color rojo claro a intenso en relación al blanquecino traslucido de otras variedades

Almendras: hace mención a que los cotiledones son morados claros.

\section{Delimitación geográfica:}

Se establece que a partir de los 1200 msnm se aceptará las peticiones a denominación de origen Cacao Arriba, manifestando que todo el territorio ecuatoriano seria permisible para tal certificación.

\section{Resultados}

La finalidad de la denominación de origen implica una serie de beneficios, pero el principal es la identificación del producto en el mercado local e internacional como impar, aportando ingresos a la balanza comercial tal es el caso de Francia con la región de Champegnoise. El empleo de una denominación de origen constituye uno de los mecanismos con los que se efectúa el proceso de diferenciación de productos en el mercado. Y unificados a herramientas como las de comercio justo identifican a productos en el mercado internacional.

Ecuador acaba de integrarse al modelo de denominación de origen en cacao fino de aroma una variedad nacional que según los expertos debe integrarse a un proceso completo que implique:

- La tipificación de plantas de variedad nacional debido a la disminución de la calidad del cacao.

- Falta de control desde el proceso de fermentado y secado del cacao en los agricultores. 
- Distribución del producto sin cuidado de la alteración de las condiciones organolépticas.

Por otra parte la falta de inversión en tecnología en la industria del cacao y chocolate, amerita que se requiera la importación de maquinaria originaria esencialmente de Italia y Estados Unidos. Considerando que Ecuador invierte apenas el 2\% del Producto Interno Bruto en investigación y tecnología. La idea de que la estrategia de denominación de origen es una herramienta de promoción.

Se evidencia una falta de planificación y control en la sección industrial; no es solo el sector del cacao un sector crítico; se denota el café, banano y camarón como fragmentos donde Ecuador debe invertir altos rubros en tecnología procedente de otros países a pesar de ser bienes altamente generadores de divisas en Ecuador.

Según La SENADI ex Instituto Ecuatoriano de la Propiedad Intelectual (IEPI) dicha denominación de origen fue conformada por un grupo interdisciplinario de varios organismos estatales con la finalidad de buscar la trazabilidad del producto y buscar actores eficientes en toda la cadena de suministro que permitan viabilizar un producto final con condiciones ideales de exportación; por lo tanto el direccionamiento de la denominación de origen está planteada para agricultores inclusive en el lenguaje y estructura.

De tal manera se puede resaltar que el interés para los organismos ecuatorianos fue identificar el logro de los agricultores para mejorar los procesos de; siembra cosecha, fermentación y secado del cacao fino de aroma para evidenciarlo así en el mercado, hasta ahora se ha conseguido un interés limitado en el sector agrícola ya que desde la propuesta, la primera denominación de origen del país Cacao Arriba; ha perfilado hasta la actualidad sesenta peticiones sin respuestas favorables y solo la adquirió una empresa agroexportadora en Santo Domingo de los Tsáchilas, a pesar de planes de socialización y de instaurar durante el año 2014 y 2015 el acceso a la denominación de origen de forma gratuita.

El sector cacaotero en el Ecuador requiere un cambio de estructura desde su raíz e involucra un proceso de mejoramiento continuo donde su vital transformación radica en la capacitación en toda la cadena de valor que incentive a los involucrados en que un producto mejor tratado en el medio en que el este interactuando generara mayores recursos , entendiéndose que el cacao actualmente se cotiza a 150 dólares el quintal se debe incentivar que este precio será más competitivo con mejoras y con cambios en su manipulación .

En el camino para lograr el cambio organizacional es necesaria la información, lo que se requiere agregar un insumo cognoscitivo para dar apoyo y explicar racionalmente los cambios propuestos y aclarar que se espera de los individuos, sin embargo, esto no constituye una fuente de motivación y se requieren otro métodos que proporcionen la fuerza motivante necesaria para el cambio (Garzón, 2005)

\section{Bibliografía}

Anecacao. (2016). Ecuador Desplaza a Camerún en Ranking mundial cacaotero. El Universo. Ayestarán Raquel, Planificación estratégica y gestión de la publicidad, Madrid, 2012 
Cavaller Víctor, Análisis de la producción científica y de patentes en el caso del tratamiento del resveratrol, QUIC\& VECT ,2008.

Cateora, P., \& Graham, J. (1999). Marketing Internacional. México: Mc Graw Hill.

Contreras, C. (1990). El sector exportador de una economía colonial.

Declaración de Denominación de origen "CACAO ARRIBA"

Díaz Márquez, Las denominaciones de origen del vino en España, 2008

Errázuriz, C. (2010). Indicaciones Geográficas y Denominaciones de Origen. Propiedad Intelectual en progreso. Revista Chilena de Derecho, 207-239.

FAO-IICA. (2008). Calidad de los alimentos vinculada al origen y las tradiciones en América Latina: estudio de casos. (Alprigrama, Ed.) Lima.

Garzón Castrillón, El desarrollo organizacional y el cambio planeado, Bogotá, 2005

Granados, L. (2004). Indicaciones geográficas y denominaciones de origen: un aporte para su implementación en Costa Rica. Costa Rica.

Ilbery, B. \& Kneafsey, M. (2000). Producer constructions of quality in regional speciality food production: a case study from south west England. Journal of Rural Studies, 16(2), 217230. doi.org/10.1016/S0743-0167 (99)00041-8

Millán Francisco, La denominación de origen: su protección jurídica, España, 2008

Porras, V. H., \& Sánchez, J. A. (1991). Enfermedades del Cacao. Honduras.

Rodríguez, G. (2004). El derecho a ostentar la denominación de origen. Las disputas por la hegemonía en el mercado agroalimentario mundial. Desacatos, 15-16. Doi: Disponible en: <http://www.scielo.org.mx/scielo.php?script=sci_arttext\&pid=S1607050X2004000200010\&lng=es\&nrm=iso>. ISSN 2448-5144

SENADI. (s/f.). Identidad Ecuatoriana en productos propios. Obtenido de Servicio nacional de Derechos Intelectuales: https://www.propiedadintelectual.gob.ec/denominacion-deorigen/

Secretaria de Economía. gob.mx/se. Obtenido de https://www.gob.mx/se/articulos/mexicocuenta-con-16-denominaciones-deorigen?idio $\mathrm{m}=\mathrm{es}$ 